\title{
A generalized mean value property for polyharmonic functions
}

\author{
Michael S. Floater*
}

December 21, 2015

\begin{abstract}
A well known property of a harmonic function in a ball is that its value at the centre equals the mean of its values on the boundary. Less well known is the more general property that its value at any point $\mathbf{x}$ equals the mean over all chords through $\mathbf{x}$ of its values at the ends of the chord, linearly interpolated at $\mathbf{x}$. In this paper we show that a similar property holds for polyharmonic functions of any order when linear interpolation is replaced by two-point Hermite interpolation of odd degree.
\end{abstract}

Math Subject Classification: Primary: 31B30, 31B10, 41A05, Secondary: 31B20, $65 \mathrm{D} 05$

Keywords: Polyharmonic functions, polynomial interpolation, integral formulas.

\section{Introduction}

Let $\Delta$ be the Laplace operator $\sum_{i=1}^{n} \partial^{2} / \partial x_{i}^{2}$ in $\mathbb{R}^{n}, n \geq 2$. A function $u$ that is $C^{2 m}$, $m \geq 1$, in a domain is said to be polyharmonic of order $m$ if $\Delta^{m} u=0$ there, where $\Delta^{k}=\Delta\left(\Delta^{k-1}\right)$. Various properties of polyharmonic functions have been developed (Almansi 1899), (Nicolesco 1935), (Aronszajn, Creese \& Lipkin 1983), (Hayman \& Korenblum 1993). In this paper we derive a new property, based on a method proposed by Gordon \& Wixom (1974) for interpolating functions in convex domains given data on the boundary.

Let $B(R)$ denote the ball in $\mathbb{R}^{n}$ of radius $R>0$,

$$
B(R)=\left\{\mathbf{x} \in \mathbb{R}^{n}:|\mathbf{x}|<R\right\},
$$

where $|\mathbf{x}|=\left(\sum_{i=1}^{n} x_{i}^{2}\right)^{1 / 2}$. The boundary of $B(R)$ is the sphere

$$
S(R)=\left\{\mathbf{y} \in \mathbb{R}^{n}:|\mathbf{y}|=R\right\} .
$$

*Department of Mathematics, University of Oslo, Moltke Moes vei 35, 0851 Oslo, Norway, email: michaelf@math.uio.no 
Suppose that $u$ is a polyharmonic function in $B(R)$ of order $m$, and that $u$ is $C^{m-1}$ in the closure $\overline{B(R)}=B(R) \cup S(R)$.

Fix some point $\mathbf{x} \in B(R)$ and let $\boldsymbol{\mu}$ be any unit vector in $\mathbb{S}^{n-1}$, the unit sphere in $\mathbb{R}^{n}$. The line through $\mathbf{x}$ in the direction $\boldsymbol{\mu}$ intersects $S(R)$ at two points,

$$
\mathbf{y}_{j}=\mathbf{x}+s_{j} \boldsymbol{\mu}, \quad j=0,1,
$$

where $s_{0}<0<s_{1}$. Specifically, using the scalar product in $\mathbb{R}^{n}$,

$$
\mathbf{y}_{j} \cdot \mathbf{y}_{j}=\left|\mathbf{y}_{j}\right|^{2}=R^{2},
$$

and we deduce that

$$
s_{j}=-\mathbf{x} \cdot \boldsymbol{\mu}-(-1)^{j} \sqrt{(\mathbf{x} \cdot \boldsymbol{\mu})^{2}+R^{2}-|\mathbf{x}|^{2}}, \quad j=0,1 .
$$

Let $p_{\boldsymbol{\mu}}(s)$ be the unique polynomial of degree $\leq M:=2 m-1$ such that

$$
\frac{d^{k}}{d s^{k}} p_{\boldsymbol{\mu}}\left(s_{j}\right)=D_{\boldsymbol{\mu}}^{k} u\left(\mathbf{y}_{j}\right), \quad k=0,1, \ldots, m-1, \quad j=0,1,
$$

where $D_{\boldsymbol{\mu}}$ denotes the directional derivative in the direction $\boldsymbol{\mu}$. We will show

\section{Theorem 1}

$$
u(\mathbf{x})=\frac{2}{\omega_{n-1}} \int_{\mathbb{H}^{n-1}} p_{\boldsymbol{\mu}}(0) d \boldsymbol{\mu},
$$

where $d \boldsymbol{\mu}$ denotes the element of $(n-1)$-dimensional area, $\omega_{n-1}$ is the surface area of $\mathbb{S}^{n-1}$, and $\mathbb{H}^{n-1}$ is any hemisphere of $\mathbb{S}^{n-1}$, for example,

$$
\mathbb{H}^{n-1}=\left\{\boldsymbol{\mu}=\left(\mu_{1}, \ldots, \mu_{n}\right) \in \mathbb{S}^{n-1}: \mu_{1} \geq 0\right\} .
$$

The harmonic case of the theorem $(m=1)$ was shown for $n=2$ in Gordon \& Wixom (1974) (attributed to W. W. Meyer) and for $n>2$ in Belyaev (2006). The biharmonic case for $n=2$ and $R=1$ was derived in Floater (2015), based on the formula of (Polyanin 2002, Sec. 9.4.1-4) for the solution to the biharmonic equation.

\section{The harmonic case}

In the harmonic case, $m=1$,

$$
p_{\boldsymbol{\mu}}(0)=\frac{s_{1} u\left(\mathbf{y}_{0}\right)-s_{0} u\left(\mathbf{y}_{1}\right)}{s_{1}-s_{0}} .
$$

Writing $\mathbf{y}_{j}=\mathbf{y}_{j, \boldsymbol{\mu}}$ and $s_{j}=s_{j, \boldsymbol{\mu}}, j=0,1$, to indicate dependence on $\boldsymbol{\mu}$, observe that $\mathbf{y}_{0, \boldsymbol{\mu}}=\mathbf{y}_{1,-\boldsymbol{\mu}}$, and $s_{j, \boldsymbol{\mu}}=-s_{1-j,-\boldsymbol{\mu}}, j=0,1$, and thus we can express the right hand side of (3) as

$$
\frac{2}{\omega_{n-1}} \int_{\mathbb{S}^{n-1}} \frac{-s_{0} u\left(\mathbf{y}_{1}\right)}{s_{1}-s_{0}} d \boldsymbol{\mu}
$$


Now $\mathbf{y}_{1}=R \boldsymbol{\nu}$ for some $\boldsymbol{\nu} \in \mathbb{S}^{n-1}$, and we change the variable of integration from $\boldsymbol{\mu}$ to $\boldsymbol{\nu}$. The relation between the $(n-1)$-dimensional area elements $d \boldsymbol{\mu}$ and $d \boldsymbol{\nu}$ is

$$
d \boldsymbol{\mu}=\frac{\cos \alpha}{\left|\mathbf{y}_{1}-\mathbf{x}\right|^{n-1}} d \mathbf{y}_{1}=\frac{R^{n-1} \cos \alpha}{\left|\mathbf{y}_{1}-\mathbf{x}\right|^{n-1}} d \boldsymbol{\nu}
$$

where $\alpha$ is the angle between $\boldsymbol{\mu}$ and $\boldsymbol{\nu}$. Since $\alpha$ is also the angle at $\mathbf{y}_{1}$ between the line segments $\left[\mathbf{y}_{1}, \mathbf{0}\right]$ and $\left[\mathbf{y}_{1}, \mathbf{x}_{0}\right]$, where $\mathbf{x}_{0}$ is the midpoint of the line segment $\left[\mathbf{y}_{0}, \mathbf{y}_{1}\right]$, we have

$$
\cos \alpha=\frac{\left|\mathbf{x}_{0}-\mathbf{y}_{1}\right|}{R}=\frac{s_{1}-s_{0}}{2 R} .
$$

Further, by the Intersecting Chords Theorem (ICT) for $\mathbf{x}$ in $B(R)$,

$$
\left(-s_{0}\right) s_{1}=\left|\mathbf{y}_{0}-\mathbf{x}\right|\left|\mathbf{y}_{1}-\mathbf{x}\right|=(R-|\mathbf{x}|)(R+|\mathbf{x}|)=R^{2}-|\mathbf{x}|^{2}
$$

(which can also be seen from (2)). Hence,

$$
-s_{0}=\left(R^{2}-|\mathbf{x}|^{2}\right) / s_{1},
$$

and substituting this and (5) and (6) into (4) shows that the right hand side of (3) is

$$
\frac{R^{n-2}\left(R^{2}-|\mathbf{x}|^{2}\right)}{\omega_{n-1}} \int_{\mathbb{S}^{n-1}} \frac{u(R \boldsymbol{\nu})}{|\mathbf{x}-R \boldsymbol{\nu}|^{n}} d \boldsymbol{\nu} .
$$

This we recognize as the Poisson integral formula for $u(\mathbf{x})$ (Gilbarg \& Trudinger 1983).

\section{Concentric spheres}

To deal with general $m$ we first establish a mean value property for $u$ with respect to concentric spheres, and show later, in Section 4, that Theorem 1 is the limiting case as the spheres coallesce.

Let $R_{1}, \ldots, R_{m}$ be any sequence of radii such that

$$
|\mathbf{x}|<R_{1}<R_{2}<\cdots<R_{m}<R .
$$

We will make use of a representation of $u$ with respect to $S\left(R_{1}\right), \ldots, S\left(R_{m}\right)$ due to Duffin \& Nehari (1961). First recall that $u$ has the unique Almansi representation

$$
u(\mathbf{x})=\sum_{l=0}^{m-1} u_{l}(\mathbf{x})|\mathbf{x}|^{2 l},
$$

for functions $u_{0} \ldots, u_{m-1}$ that are harmonic in $B(R)$; see (Almansi 1899), (Nicolesco 1936), and (Aronszajn et al. 1983, Chap. 1). By Lagrange polynomial interpolation of degree $m-1$,

$$
|\mathbf{x}|^{2 l}=\sum_{j=1}^{m} R_{j}^{2 l} \prod_{\substack{k=1 \\ k \neq j}}^{m} \frac{|\mathbf{x}|^{2}-R_{k}^{2}}{R_{j}^{2}-R_{k}^{2}},
$$


and substituting this into (9) gives the representation of $u$ of (Duffin \& Nehari 1961),

$$
u(\mathbf{x})=\sum_{j=1}^{m} v\left(\mathbf{x} ; R_{j}\right) \prod_{\substack{k=1 \\ k \neq j}}^{m} \frac{|\mathbf{x}|^{2}-R_{k}^{2}}{R_{j}^{2}-R_{k}^{2}},
$$

where, for any $\rho, 0<\rho<R$,

$$
v(\mathbf{x} ; \rho):=\sum_{l=0}^{m-1} u_{l}(\mathbf{x}) \rho^{2 l} .
$$

Observe that $v(\cdot, \rho)$ is harmonic for each $\rho$, and, comparing (11) with (9), we see that if $|\mathbf{x}|=R_{j}$, then $v\left(\mathbf{x}, R_{j}\right)=u(\mathbf{x})$, i.e., $v\left(\cdot, R_{j}\right)$ agrees with $u$ on the sphere $S\left(R_{j}\right)$.

Consider now, as in Section 2, the line in the direction $\boldsymbol{\mu} \in \mathbb{S}^{n-1}$ through $\mathbf{x}$. It intersects the spheres $S\left(R_{1}\right), \ldots, S\left(R_{m}\right)$ at the $2 m$ points

$$
\mathbf{z}_{j}=\mathbf{x}+t_{j} \boldsymbol{\mu}, \quad-m+1 \leq j \leq m,
$$

where

$$
t_{-m+1}<\cdots<t_{0}<0<t_{1}<\cdots<t_{m} .
$$

The intersections with $S\left(R_{j}\right)$ are $\mathbf{z}_{1-j}$ and $\mathbf{z}_{j}, j=1, \ldots, m$. Let $q_{\boldsymbol{\mu}}(s)$ be the polynomial of degree $\leq M$ such that

$$
q_{\boldsymbol{\mu}}\left(t_{j}\right)=u\left(\mathbf{z}_{j}\right), \quad-m+1 \leq j \leq m .
$$

Using the representation (10) we will show

\section{Lemma 1}

$$
u(\mathbf{x})=\frac{2}{\omega_{n-1}} \int_{\mathbb{H}^{n-1}} q_{\boldsymbol{\mu}}(0) d \boldsymbol{\mu} .
$$

Proof. From the Lagrange form of $q_{\boldsymbol{\mu}}(s)$,

$$
q_{\boldsymbol{\mu}}(0)=\sum_{j=-m+1}^{m} L_{j} u\left(\mathbf{z}_{j}\right),
$$

where

$$
L_{j}=\prod_{\substack{k=-m+1 \\ k \neq j}}^{m} \frac{-t_{k}}{t_{j}-t_{k}} .
$$

In analogy to the derivation of (4), writing $L_{j}=L_{j, \boldsymbol{\mu}}$ to indicate its $\boldsymbol{\mu}$-dependence, we find $L_{1-j, \mu}=L_{j,-\mu}$, and therefore the right hand side of (12) is

$$
\frac{2}{\omega_{n-1}} \int_{\mathbb{S}^{n-1}} \sum_{j=1}^{m} L_{j} u\left(\mathbf{z}_{j}\right) d \boldsymbol{\mu} .
$$


For $j=1, \ldots, m$, we can express $L_{j}$ as

$$
L_{j}=\frac{-t_{1-j}}{t_{j}-t_{1-j}} \prod_{\substack{k=1 \\ k \neq j}}^{m} \frac{t_{k} t_{1-k}}{\left(t_{j}-t_{k}\right)\left(t_{j}-t_{1-k}\right)} .
$$

By ICT for $\mathbf{x}$ in $B\left(R_{k}\right)$ we have

$$
t_{k} t_{1-k}=-\left|\mathbf{z}_{k}-\mathbf{x}\right|\left|\mathbf{z}_{1-k}-\mathbf{x}\right|=|\mathbf{x}|^{2}-R_{k}^{2} .
$$

For $k>j$ in (14), by ICT for $\mathbf{z}_{j}$ in $B\left(R_{k}\right)$,

$$
\left(t_{j}-t_{k}\right)\left(t_{j}-t_{1-k}\right)=-\left|\mathbf{z}_{k}-\mathbf{z}_{j}\right|\left|\mathbf{z}_{j}-\mathbf{z}_{1-k}\right|=R_{j}^{2}-R_{k}^{2} .
$$

For $k<j$ in (14), by symmetry,

$$
t_{k}+t_{1-k}=t_{j}+t_{1-j}
$$

and so by ICT for $\mathbf{z}_{k}$ in $B\left(R_{j}\right)$,

$$
\left(t_{j}-t_{k}\right)\left(t_{j}-t_{1-k}\right)=\left(t_{j}-t_{k}\right)\left(t_{k}-t_{1-j}\right)=\left|\mathbf{z}_{j}-\mathbf{z}_{k}\right|\left|\mathbf{z}_{k}-\mathbf{z}_{1-j}\right|=R_{j}^{2}-R_{k}^{2},
$$

again. Thus,

$$
L_{j}=\frac{-t_{1-j}}{t_{j}-t_{1-j}} \prod_{\substack{k=1 \\ k \neq j}}^{m} \frac{|\mathbf{x}|^{2}-R_{k}^{2}}{R_{j}^{2}-R_{k}^{2}} .
$$

Substituting this into (13), and since $v\left(\mathbf{x} ; R_{j}\right)$ can be expressed as (4) with $R=R_{j}$, we find that (13) equals the right hand side of (10), which equals $u(\mathbf{x})$.

\section{Proof of Theorem 1}

To prove Theorem 1 from Lemma 1 it remains to show that

$$
\int_{\mathbb{H}^{n-1}} q_{\boldsymbol{\mu}}(0) d \boldsymbol{\mu} \rightarrow \int_{\mathbb{H}^{n-1}} p_{\boldsymbol{\mu}}(0) d \boldsymbol{\mu}
$$

as $R_{1}, \ldots, R_{m} \rightarrow R$, under the assumption that $u$ is $C^{m-1}$ in $\overline{B(R)}$. The convergence of (15) is equivalent to

$$
\int_{\mathbb{H}^{n-1}}\left(u(\mathbf{x})-q_{\boldsymbol{\mu}}(0)\right) d \boldsymbol{\mu} \quad \rightarrow \quad \int_{\mathbb{H}^{n-1}}\left(u(\mathbf{x})-p_{\boldsymbol{\mu}}(0)\right) d \boldsymbol{\mu} .
$$

Fix $\boldsymbol{\mu} \in \mathbb{H}^{n-1}$. By the Newton error formula for Lagrange and Hermite interpolation, if $u_{\boldsymbol{\mu}}(s):=u(\mathbf{x}+s \boldsymbol{\mu}), s_{0} \leq s \leq s_{1}$, then $u_{\boldsymbol{\mu}} \in C^{m-1}\left[s_{0}, s_{1}\right]$ and

$$
\begin{aligned}
& u(\mathbf{x})-p_{\boldsymbol{\mu}}(0)=u_{\boldsymbol{\mu}}(0)-p_{\boldsymbol{\mu}}(0)=s_{0}^{m} s_{1}^{m}[\underbrace{s_{0}, \ldots, s_{0}}_{m}, \underbrace{s_{1}, \ldots, s_{1}}_{m}, 0] u_{\boldsymbol{\mu}}, \\
& u(\mathbf{x})-q_{\boldsymbol{\mu}}(0)=u_{\boldsymbol{\mu}}(0)-q_{\boldsymbol{\mu}}(0)=t_{-m+1} \cdots t_{m}\left[t_{-m+1}, \ldots, t_{m}, 0\right] u_{\boldsymbol{\mu}} .
\end{aligned}
$$


If $R_{1}, \ldots, R_{m} \rightarrow R$, then $t_{-m+1}, \ldots, t_{0} \rightarrow s_{0}$ and $t_{1}, \ldots, t_{m} \rightarrow s_{1}$ and, by the theory of divided differences (Isaacson \& Keller 1994), (Conte \& de Boor 1980),

$$
\left[t_{-m+1}, \ldots, t_{m}, 0\right] u_{\boldsymbol{\mu}} \rightarrow[\underbrace{s_{0}, \ldots, s_{0}}_{m}, \underbrace{s_{1}, \ldots, s_{1}}_{m}, 0] u_{\boldsymbol{\mu}},
$$

and it follows from (17) that $u(\mathbf{x})-q_{\boldsymbol{\mu}}(0) \rightarrow u(\mathbf{x})-p_{\boldsymbol{\mu}}(0)$. Moreover, for $R_{1}, \ldots, R_{m}$ close enough to $R$, it follows from (17) that $\left|u(\mathbf{x})-q_{\boldsymbol{\mu}}(0)\right|$ is bounded by a constant, independent of $\boldsymbol{\mu} \in \mathbb{H}^{n-1}$, and this proves the convergence of (16) and hence Theorem 1.

\section{Boundary derivatives}

To express the integral formula (3) in terms of the boundary derivatives of $u$, we can first express the Hermite polynomial $p_{\boldsymbol{\mu}}$ in Bernstein form (Prautzsch, Boehm \& Paluszny 2002, Sec. 4.4) as

$$
p_{\boldsymbol{\mu}}(s)=\sum_{j=0}^{M} b_{j} B_{j}^{M}(\lambda),
$$

where $\lambda=\left(s-s_{0}\right) /\left(s_{1}-s_{0}\right)$, and

$$
B_{j}^{M}(\lambda)=\left(\begin{array}{c}
M \\
j
\end{array}\right) \lambda^{j}(1-\lambda)^{M-j},
$$

and

$$
\begin{aligned}
b_{j} & =\sum_{k=0}^{j}\left(\begin{array}{l}
j \\
k
\end{array}\right) \frac{(M-k) !}{M !}\left(s_{1}-s_{0}\right)^{k} D_{\mu}^{k} u\left(\mathbf{y}_{0}\right), \\
b_{M-j} & =\sum_{k=0}^{j}(-1)^{k}\left(\begin{array}{l}
j \\
k
\end{array}\right) \frac{(M-k) !}{M !}\left(s_{1}-s_{0}\right)^{k} D_{\boldsymbol{\mu}}^{k} u\left(\mathbf{y}_{1}\right),
\end{aligned}
$$

for $j=0,1, \ldots, m-1$. From this we find

$$
p_{\boldsymbol{\mu}}(s)=\sum_{j=0}^{m-1} \frac{1}{j !}\left(s_{1}-s_{0}\right)^{j}\left(a_{j, m}(\lambda) D_{\boldsymbol{\mu}}^{j} u\left(\mathbf{y}_{0}\right)+(-1)^{j} a_{j, m}(1-\lambda) D_{\boldsymbol{\mu}}^{j} u\left(\mathbf{y}_{1}\right)\right),
$$

where

$$
a_{j, m}(\lambda)=\sum_{i=0}^{m-1-j}\left(\begin{array}{c}
M-j \\
i
\end{array}\right) \lambda^{i+j}(1-\lambda)^{M-i-j} .
$$

Then, similar to the derivation of (4) and (13), we obtain a more explicit form of (3):

\section{Corollary 1}

$$
u(\mathbf{x})=\sum_{j=0}^{m-1} \frac{(-1)^{j}}{j !} \frac{2}{\omega_{n-1}} \int_{\mathbb{S}^{n-1}}\left(s_{1}-s_{0}\right)^{j} a_{j, m}\left(\frac{s_{1}}{s_{1}-s_{0}}\right) D_{\boldsymbol{\mu}}^{j} u\left(\mathbf{y}_{1}\right) d \boldsymbol{\mu} .
$$


From this we obtain an apparently new mean value property of $u$ at the centre of the ball $B(R)$ by specializing to $\mathbf{x}=\mathbf{0}$. In that case, $s_{1}=R, s_{0}=-R$, and $\mathbf{y}_{1}=R \boldsymbol{\mu}$, and we deduce

\section{Corollary 2}

$$
u(\mathbf{0})=\frac{1}{4^{m-1}} \sum_{j=0}^{m-1} c_{j, m} \frac{(-2 R)^{j}}{j ! \omega_{n-1}} \int_{\mathbb{S}^{n-1}} D_{\boldsymbol{\mu}}^{j} u(R \boldsymbol{\mu}) d \boldsymbol{\mu},
$$

where $c_{j, m}$ is the partial sum of binomial coefficients,

$$
c_{j, m}=\sum_{i=0}^{m-1-j}\left(\begin{array}{c}
M-j \\
i
\end{array}\right) .
$$

The first few cases of the coefficients $c_{j, m}$ are

$$
\begin{aligned}
c_{0,1} & =1, \\
\left(c_{0,2}, c_{1,2}\right) & =(4,1), \\
\left(c_{0,3}, c_{1,3}, c_{2,3}\right) & =(16,5,1) \\
\left(c_{0,4}, c_{1,4}, c_{2,4}, c_{3,4}\right) & =(64,22,6,1) .
\end{aligned}
$$

\section{Poisson-type integral}

It is interesting to compare the integral formula (3) with the Poisson-type integral formula derived by (Duffin \& Nehari 1961) and (Edenhoffer 1975). Equation (10) expresses $u(\mathbf{x})$ as the value at $|\mathbf{x}|^{2}$ of the Lagrange polynomial interpolant of degree $\leq m-1$ at the points $R_{j}^{2}$ to the values $v\left(\mathbf{x} ; R_{j}\right), j=1, \ldots, m$. Therefore, taking the limit as $R_{1}, \ldots, R_{m} \rightarrow R$ makes $u(\mathbf{x})$ the Taylor series,

$$
u(\mathbf{x})=\sum_{i=0}^{m-1} \frac{1}{i !}\left(|\mathbf{x}|^{2}-R^{2}\right)^{i} \frac{\partial^{i}}{\partial\left(R^{2}\right)^{i}} v(\mathbf{x} ; R) .
$$

By the Leibniz rule applied to the product of $1 /\left(|\mathbf{x}|^{2}-R^{2}\right)$ and $v(\mathbf{x} ; R)$, we can rewrite this as

$$
u(\mathbf{x})=\frac{\left(|\mathbf{x}|^{2}-R^{2}\right)^{m}}{(m-1) !} \frac{\partial^{m-1}}{\partial\left(R^{2}\right)^{m-1}}\left(\frac{v(\mathbf{x} ; R)}{|\mathbf{x}|^{2}-R^{2}}\right) .
$$

Applying the Poisson formula (8) to $v$ now gives

$$
u(\mathbf{x})=\frac{-\left(|\mathbf{x}|^{2}-R^{2}\right)^{m}}{(m-1) ! \omega_{n-1}} \int_{\mathbb{S}^{n-1}} \frac{\partial^{m-1}}{\partial\left(R^{2}\right)^{m-1}}\left(\frac{R^{n-2} u(R \boldsymbol{\nu})}{|\mathbf{x}-R \boldsymbol{\nu}|^{n}}\right) d \boldsymbol{\nu},
$$

as found by (Duffin \& Nehari 1961) and (Edenhoffer 1975).

Unlike (3), this formula requires repeated differentiation in the integrand with respect to $R^{2}$, and it would be more difficult to derive Corollary 2 from this for general $m$. One might also try to prove Theorem 1 by transforming (3) into (20). In the special case that $n=2$ and $m=2$, this was the approach used in (Floater 2015), based on expressing the first directional derivative with respect to $\boldsymbol{\mu}$ at a point on the sphere as a linear combination of a normal and tangent derivative. This approach, however, does not look promising when either $m \geq 3$ or $n \geq 3$. 


\section{$7 \quad$ Final remarks}

- Various numerical integration methods can be used to evaluate the integral in (3) and to verify Theorem 1 and Corollaries 1 and 2 numerically. A simple choice in the planar case $(n=2)$ is to let $\boldsymbol{\mu}=(\cos \theta, \sin \theta)$ and use the periodic trapezoidal rule with respect to the angle $\theta$, as suggested in (Gordon \& Wixom 1974).

- The linear-based method of (Gordon \& Wixom 1974) $(m=1)$ for general convex domains has recently gained interest as a method of constructing generalized barycentric coordinates (GBCs) in polygonal domains (Belyaev 2006), (Manson, Li \& Schaefer 2011), (Li \& Hu 2013), (Li, Ju \& Hu 2013), (Floater 2015). GBCs have various applications in graphics and geometric modelling, such as surface parameterization and the deformation of images and shapes. Although Gordon-Wixom coordinates do not have a simple closed form like Wachspress and mean values coordinates for example, they have the advantage of being 'pseudo-harmonic', i.e., they are close to harmonic when the polygon is close to a circle. This paper shows that Gordon-Wixom coordinates of any order $m$ are similarly 'pseudo-polyharmonic'. This means that, at least for convex domains, Gordon-Wixom coordinates of general order could be considered as a practical alternative to polyharmonic coordinates: requiring less computational effort, but approximating the polyharmonic ones. For shape deformation, the advantage of using biharmonic coordinates instead of harmonic ones was recently demonstrated in (Weber, Poranne \& Gotsman 2012), and in that case one might consider the alternative of Gordon-Wixom coordinates of order $m=2$.

Acknowledgement. I wish to thank the two referees and the editor for their careful reading of the manuscript and their valuable comments which have helped to improve the paper.

\section{References}

Almansi, E. (1899), 'Sulle integrazione dell'equazione differenciale $\Delta^{2 n}=0$ ', Annali di Matematica 2, 1-59.

Aronszajn, N., Creese, T. \& Lipkin, L. (1983), Polyharmonic functions, Clarendon Press, Oxford.

Belyaev, A. (2006), On transfinite barycentric coordinates, in 'Symposium on Geometry Processing 2006', Eurographics Association, pp. 89-99.

Conte, S. D. \& de Boor, C. (1980), Elementary numerical analysis, McGraw-Hill.

Duffin, R. J. \& Nehari, Z. (1961), 'Note on polyharmonic functions', Proc. Amer. Math. Soc. 12, 110-115.

Edenhoffer, J. (1975), 'Integraldarstellungen einer $m$-polyharmonischen funktion, deren funktionswerte und erste $m-1$ normalableitungen auf einer hypersphäre gegeben sind', Math. Nachr. 68, 105-113. 
Floater, M. S. (2015), 'Generalized barycentric coordinates and applications', Acta Numerica 24, 161-214.

Gilbarg, D. \& Trudinger, N. S. (1983), Elliptic partial differential equations of second order, Springer, Berlin.

Gordon, W. J. \& Wixom, J. A. (1974), 'Pseudo-harmonic interpolation on convex domains', SIAM J. Numer. Anal. 11, 909-933.

Hayman, W. K. \& Korenblum, B. (1993), 'Representation and uniqueness theorems for polyharmonic functions', J. d'Analyse Math. 60, 113-133.

Isaacson, E. \& Keller, H. B. (1994), Analysis of numerical methods, Dover.

Li, X.-Y. \& Hu, S.-M. (2013), 'Poisson coordinates', IEEE Trans. Vis. Comput. Graph. 19, 344-352.

Li, X.-Y., Ju, T. \& Hu, S.-M. (2013), 'Cubic mean value coordinates', ACM Trans. on Graph. 32, 126:1-126:10.

Manson, J., Li, K. \& Schaefer, S. (2011), 'Positive Gordon-Wixom coordinates', Computer Aided Design 43, 1422-1426.

Nicolesco, M. (1935), 'Nouvelles contributions dans la théorie des fonctions polyharmoniques', Bull. Math. Soc. Sei. Math. Phys. R.P. Roumaine 37, 79-95.

Nicolesco, M. (1936), 'Les fonctions polyharmoniques', Actualités scientifiques et industrielles 331.

Polyanin, A. D. (2002), Handbook of linear partial differential equations for engineers and scientists, Chapman and Hall.

Prautzsch, H., Boehm, W. \& Paluszny, M. (2002), Bézier and B-spline techniques, Springer.

Weber, O., Poranne, R. \& Gotsman, C. (2012), 'Biharmonic coordinates', Comput. Graph. Forum 31, 2409-2422. 